\title{
Advanced EBSD Pattern Interpretation through Iterative Post-Processing
}

\author{
Gert Nolze $^{1}$, Eric Payton ${ }^{1}$ and Aimo Winkelmann ${ }^{2}$ \\ 1. Federal Institute for Materials Research and Testing (BAM), Division 5.1, Berlin, Germany \\ 2. Max-Planck Institut für Mikrostrukturphysik, Halle, Germany
}

Electron backscatter diffraction (EBSD) in the scanning electron microscope (SEM) is nowadays a widely accepted tool for locally resolved crystal orientation investigations and microstructure characterization. Although the original backscattered signal is discarded in many cases, some analytical systems use pattern streaming to keep each diffraction pattern either temporarily (for compatibility between higher acquisition speed and slower indexing speeds), or permanently (to enable offline indexing of patterns) [1]. Commercially available multicore computers generally determine orientations faster than current cameras can capture and transfer sufficient quality diffraction images from the phosphor screen, even when the images are transferred in a highly binned mode. Despite the fact that the diffraction signal contains more information than just the band positions for orientation measurements, only a background-corrected signal is generally used. By interpreting the EBSD screen as an array of multiple detectors, both Wells et al. [2] and Schwarzer et al. [3] have showed that the pattern images contain topographic, composition, and orientation contrast, and that the relative amounts of each contrast source varies with the pixel location within the array. Deriving such a EBSD-BSE signal from the patterns themselves has certain advantages in comparison to semiconductor detectors typically positioned around the EBSD camera screen: The images are less noisy, the image resolution is sharper due to energy filtering from the Al-coated phosphor screen - cf. Fig. 1 - and the size and position of the detector array may be freely adapted in order to increase or decrease the effects of topography and orientation contrast on the image. Since the BSE signal depends on many factors, like the chemistry of the phase and the acceleration voltage, the size and position of the detector array is (slightly) different from phase to phase so that an (iterative) post-processing of the stored patterns is highly recommended. The derived BSE signal can be used for phase assignment in high resolution and high speed maps when EBSD fails and/or EDS (energy dispersive spectroscopy) needs too much time for a suitable and parallel signal acquisition.

An iterative processing of diffraction patterns is not only beneficial for the background signal but also for the commonly used and already processed modulated diffraction signal. Modern detectors are equipped with cameras which are able to transfer more high resolution images than the typical 80x60 or 160x120 pixel images generally used in EBSD. The real limiting factor is the Hough transformation, which is computationally intensive and often limits the practical pattern size due to the time required to process the large number of patterns collected in a typical EBSD scan. In effect, the potential angular resolution benefits of collecting EBSD images at high spatial resolutions are lost due to downsampling the image prior to the Hough transformation. One potential solution to this problem is to apply a local increase in the Hough resolution after identifying the peaks in the standard low-resolution Hough transform of the image. This iterative post-processing can enable a more accurate determination of band locations, which can then be used for either improved system calibration or orientation determination. Furthermore, it can result in a more precise band width approximation as well as better quality band profiles, which can then be used for more accurate phase identification, e.g. in combination with dynamically simulated EBSD patterns [4]. 
Another area where advanced EBSD pattern processing on higher-resolution images may be beneficial is in band detection using image convolutions, such as the static butterfly filter. Gnomonic distortions systematically increase with distance from the pattern center. When the pattern center is far away from the image center, this results in difficulties determining band positions. A phase-specific optimization of the image convolution filter can be employed, taking the lattice parameters, the pattern center, the acceleration voltage, etc. into account. Such iterative post-processing of EBSD data could result in more accurate phase and orientation determinations.

In summary, pattern streaming is obviously not only a nice feature for displaying the experimental signal at each position and for a post-indexing of phases in a sample that were unknown at the time of the scan, it is a basic requirement for real post-processing of measured data, allowing for iterative improvements of angular accuracy and phase identification reliability.

\section{References:}

[1] R.A. Schwarzer and J. Hjelen, Solid State Phenomena 160 (2010), p.295.

[2] O.C. Wells et al, Scanning 28 (2006), p. 27.

[3] J. R. Schwarzer, J. Sukkau and J. Hjelen. Microscopy Conference (2011), Kiel, M.P007

[4] A. Winkelmann et al., Ultramicroscopy 107 (2007), p.414

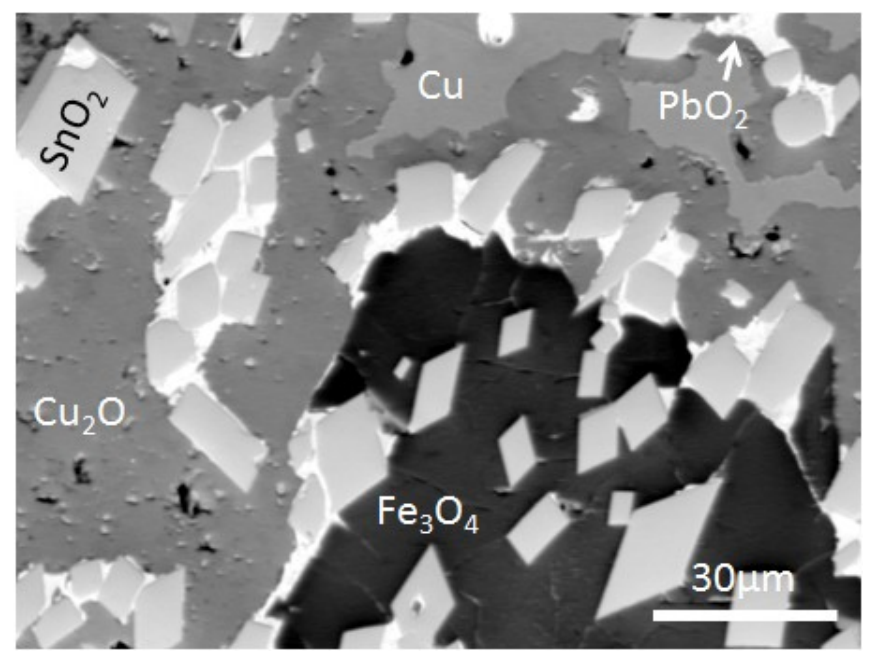

BSE detector image

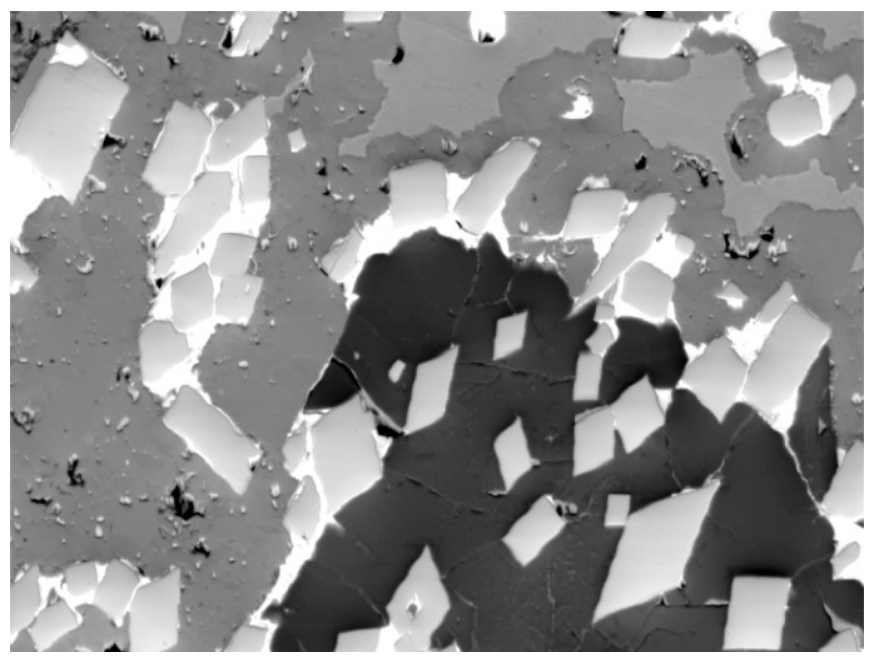

EBSD-BSE image

Figure 1. Comparison between the BSE image of a $\mathrm{Cu}$ dross collected by a BSE detector above the EBSD screen (left), and a BSE image derived from EBSD patterns (right). 